Check for updates

Cite this: RSC Adv., 2019, 9, 16967

\title{
Small molecule PROTACs: an emerging technology for targeted therapy in drug discovery
}

\author{
Haixiang Pei, ${ }^{a}$ Yangrui Peng, (D) ${ }^{a}$ Qiuhua Zhao*b and Yihua Chen (D) *a
}

Curing malignant carcinomas is a grand ambition in the development of human health. Over the past decades, targeted therapies have become one of the most successful ways of achieving this. Of these approaches, small molecule inhibitors and monoclonal antibodies are two major methods, however several barriers to their development and clinical use still exist. The use of proteolysis-targeting chimeras (PROTACs) is a new technology through utilizing a intracellular ubiquitin-proteasome system to induce targeted protein degradation, is receiving much attention in the field of targeted therapies. Heterobifunctional PROTACs have the potential to eliminate the "undruggable" proteome that comprises about $85 \%$ of human proteins, which indicates their great prospects in therapeutic fields. However, there are some hurdles preventing current PROTACs moving from bench to clinic, such as delivery and bioavailability. This review provides an overview of the development of PROTAC technology and will briefly summarize the future possible directions of this approach.

Received 7th May 2019

Accepted 14th May 2019

DOI: $10.1039 / c 9 r a 03423 d$

rsc.li/rsc-advances always cause off-target effects. ${ }^{2}$ Thirdly, continuous exposure to small molecule inhibitors always induces the mutation of target proteins, which will cause drug-resistance. ${ }^{3}$ In addition, the compensatory feedback activation of downstream signaling may happen when inhibiting only one specific target., ${ }^{\mathbf{4} 5}$ Moreover, the long-term inhibition of target proteins may lead to compensatory protein overexpression and intracellular protein accumulation, which may lead to incomplete inhibition, and thus an increase in drug dosages. ${ }^{6}$ All these limitations seriously obstruct the discovery and durable clinical effectiveness of small molecule inhibitors.

A novel concept called induced protein degradation has the potential to overcome most of the limitations of small molecule inhibitors. This strategy proposes that a small molecule just needs a brief interaction with its target protein, leading to the loss of function of the target. Compared to the "occupancydriven" mode of small molecule inhibitors, this "event-driven" pharmacology leads the target protein to attain loss-of-function via a transient binding event, which is based on employing the cellular ubiquitin-proteasome system (UPS) to induce target protein ubiquitination for destructive purposes ${ }^{7}$. After targetprotein destruction, this small-molecule drug can survive and carry on another cycle of target-protein degradation (Fig. 1). This sub-stoichiometric activity is catalytic in nature, avoiding maintaining a high level of drug dosage. ${ }^{8}$ As the target protein must be resynthesized, the overexpression and accumulation of the target protein can be averted. Theoretically, this approach has the potential to target the "undruggable" proteome that limits traditional drugs, as the warhead needs only slight binding affinity to recruit the protein of interest (POI) rather than high inhibition activity. ${ }^{9}$ HSP inhibitors and hydrophobic
${ }^{a}$ Shanghai Key Laboratory of Regulatory Biology, The Institute of Biomedical Sciences, School of Life Sciences, East China Normal University, Shanghai 200241, China. E-mail:yhchen@bio.ecnu.edu.cn

${ }^{b}$ School of Chemistry and Molecular Engineering, East China Normal University, Shanghai 200241, China.E-mail: qhzhao@chem.ecnu.edu.cn 


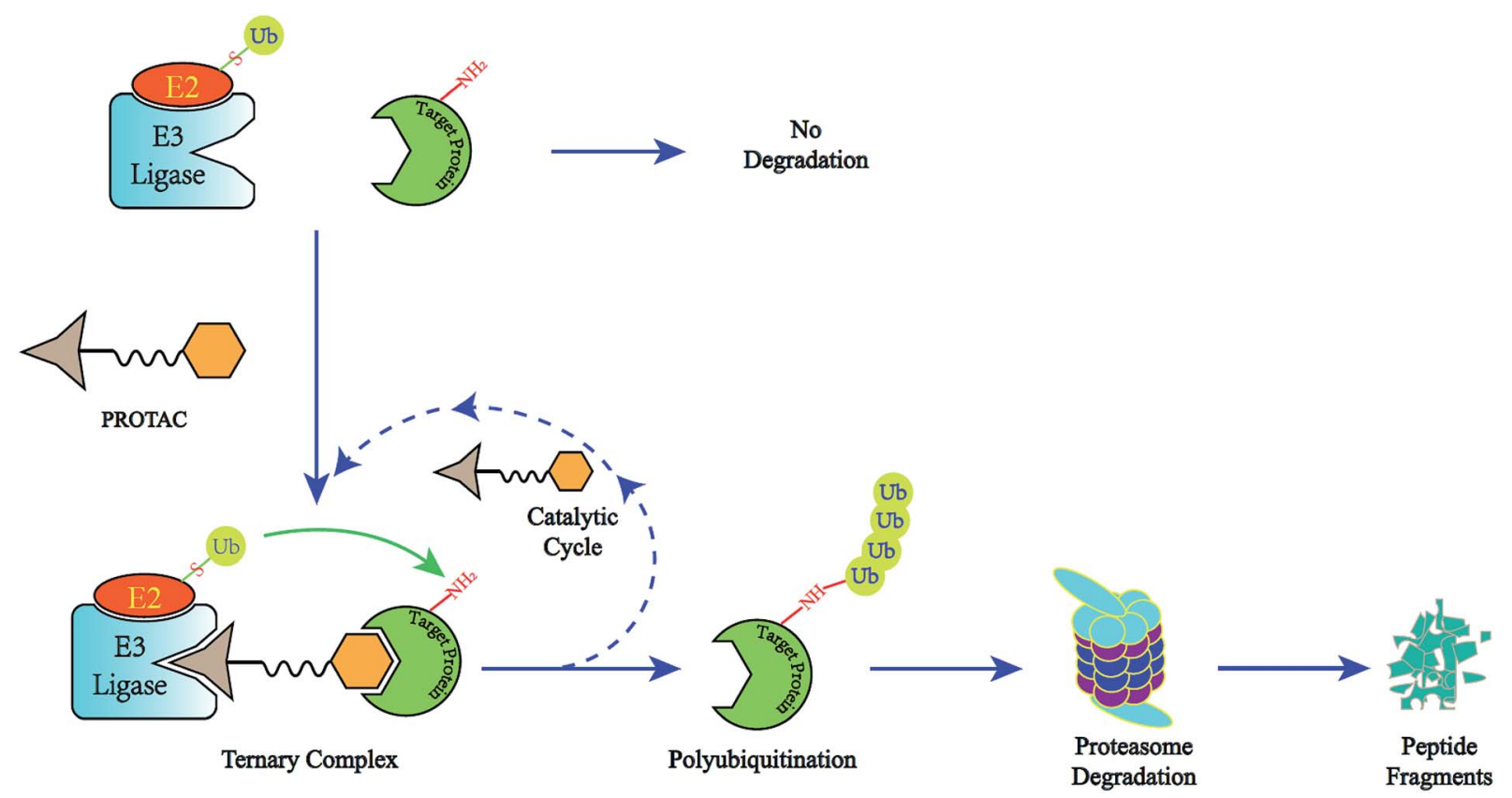

Fig. 1 The degradation cycle of an E3 ligase using PROTACs. The blue oval represents the protein of interest (POI) and the orange oval represents the E3 ligase for recruiting. A PROTAC molecule comprises a warhead to target the POI, an E3 ligand to recruit the E3 ligase and a linker to connect them. When the PROTAC molecule draws the POI and E3 ligase close, E3 will employ an E2 ubiquitin-conjugating enzyme to transfer ubiquitin to the surface of the targeted protein. Then the proteasome will recognize the polyubiquitination signal and degrade the POI. At the same time, the PROTAC molecule will separate from the ternary complex and participate in another degradation cycle.

tagging strategies were the first generation of drugs targeting protein degradation, however poor druggability and pharmacological defects impeded the clinical application of these methods. $^{7}$

The use of proteolysis-targeting chimeras (PROTACs) is a new area of induced protein degradation, which employs hetero-bifunctional molecules that induce a ligand to bind with the target protein, another ligand to recruit an E3 ubiquitin ligase, and a linker to concatenate the two ligands. Once the ternary complex (target-PROTAC-E3) is formed, the recruited E3 will employ an E2 ubiquitin-conjugating enzyme to transfer ubiquitin to the surface of the targeted protein. A polyubiquitination signal will be recognized by the proteasome to promote the degradation of the targeted proteins. To date, PROTAC technology has been used to induce the degradation of various proteins, including kinases, skeleton proteins, nuclear receptors and transcriptional factors, as well as regulatory proteins. In recent years, this technology has drawn much attention from researchers worldwide in different fields. According to data from Web of Science, over 200 papers have been reported from 2001; a growth spurt was observed in the past five years, especially (Fig. 2). Also, PROTAC technology holds great promise for development for clinic use. Several companies, such as Arvinas, C4 Therapeutics, Kymera Therapeutics and Captor Therapeutics, are setting about pushing the development of PROTAC. The pioneer Arvinas leads the way and is making great efforts to push its two PROTAC molecules into clinic trails. According to the latest news, the FDA has cleared an application from Arvinas for phase I trials of the oral PROTAC ARV-110 drug, which targets the androgen receptor in metastatic castration-resistant prostate cancer. These encouraging superiorities have aroused much interest from many big pharmaceutical companies. Arvinas has announced research collaboration and a licensing agreement with Pfizer and obtained over one hundred million dollars of financing to advance PROTAC technology. C4 therapeutics has gained a strategic collaboration with Roche and launched with 73 million dollars of series A financing.

This review will summarize the development of PROTAC technology. We place emphasis on elaborating the advantages of small-molecule PROTACs compared to small molecule inhibitors, together with the associated challenges and potential development directions. In addition, we also analyze deficiencies and forecast the future development of PROTACs.

\section{PROTAC technology}

\subsection{Peptide-based PROTACs}

In 2001, Crews and his colleagues put forward the concept of PROTACs for the first time. ${ }^{10}$ The first-generation PROTACs contain a phosphopeptide that binds to the E3 ligase $\beta$-TRCP, and a small-molecule Ovalicin that targets MetAP-2. The lack of cell permeability of the peptide moieties is the major defect of this series of PROTACs, which limits their utility as chemical probes. The first cell-permeable PROTACs, coupling a poly-D-Arg sequence to a peptide derived from HIF- $1 \alpha$ that binds to the VHL (von Hippel Lindau) E3 ligase, were developed in 2004. ${ }^{11}$ These peptide-based PROTACs showed the efficient degradation of targets in cultured cells (e.g., FKBP12 ${ }^{\mathrm{F} 36 \mathrm{~V}}$, MetAP-2, and androgen and estrogen receptors). Despite many efforts having 


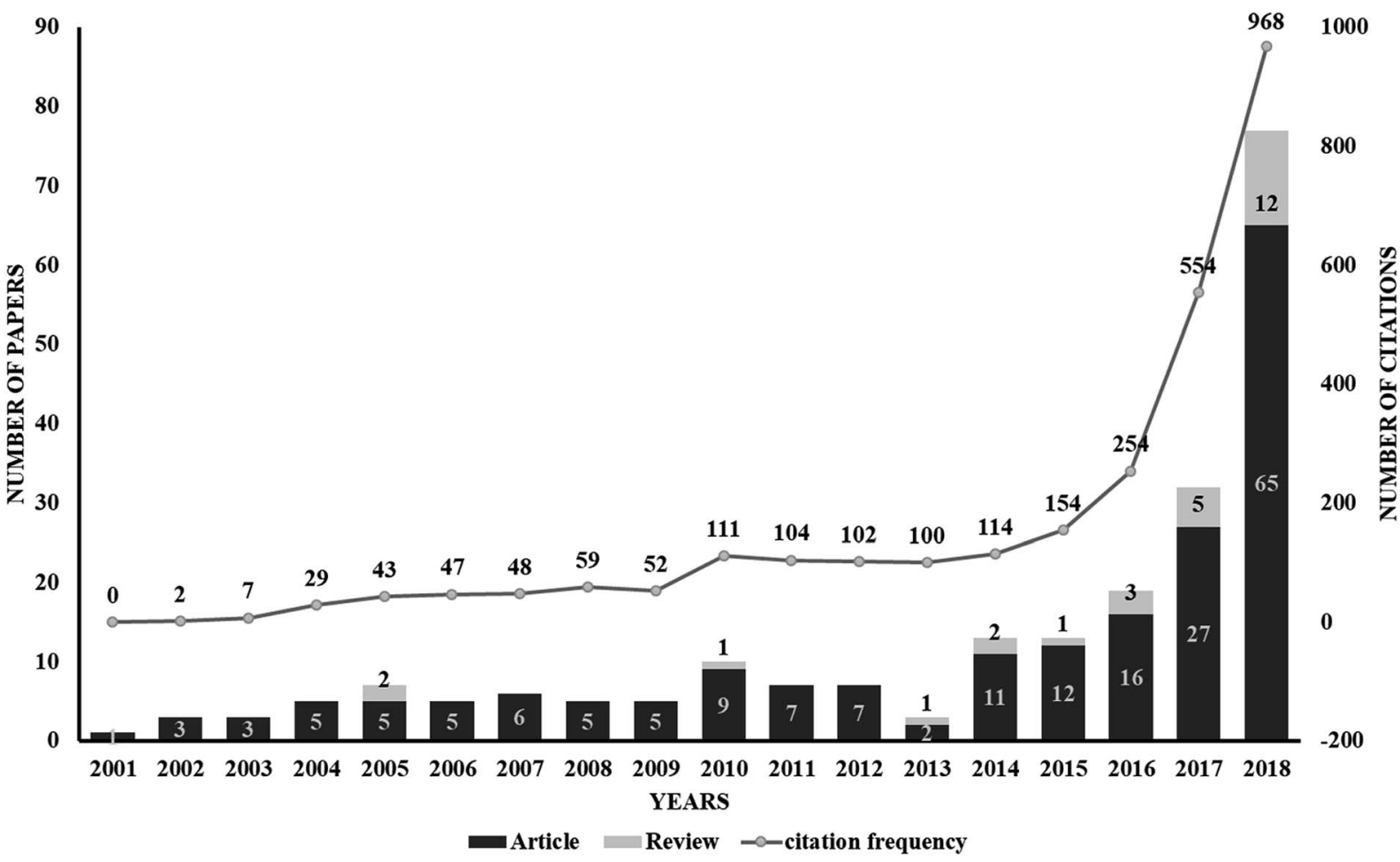

Fig. 2 A graph showing the publications associated with PROTAC technology. Data from Web of Science (http://apps.webofknowledge.com). This graph reflects the number of articles and reviews. As can be seen, a drastic increase from 2014 indicates the rapid development of PROTAC technology

been made to optimize peptide-based PROTACs, inherent defects such as high molecular weight, low potency, poor cell permeability and peptide bond lability restrict the potential clinical use of peptide-based PROTACs.

\subsection{Small-molecule PROTACs}

Although many shortcomings exist, the first-generation peptidebased PROTACs proved that recruiting the E3 ligase to induce protein degradation is a feasible strategy to generate a new direction for drug development. All-small-molecule PROTACs have been proposed to achieve better cell permeability and rapid target degradation. So far, with the successful development of small-molecule ligands, four E3 ligases (MDM2, cIAP1, CRBN (cereblon) and von Hippel-Lindau (VHL)) have been used as the basis for all-small-molecule PROTACs to selectively degrade target proteins.
2.2.1 MDM2-based PROTAC. The first all-small-molecule PROTAC, which couples nutlin to an androgen receptor (AR) ligand employing the MDM2 E3 ligase, induced the degradation of AR in prostate tumor cells. ${ }^{\mathbf{1 2}}$ Although this PROTAC molecule is less effective at degrading AR than its peptide analogues, it provides proof-of-concept that an all-small-molecule PROTAC is practicable and displays the potential of PROTAC technology as a new therapy method for various diseases (Fig. 3).

2.2.2 IAP-based PROTACs. In 2010, Y. Hashimoto and his group successfully connected bestatin esters with all-trans retinoic acid with different length linkers. ${ }^{13}$ These PROTACs can utilize cellular inhibitor of apoptosis protein 1 (cIAP1) to induce the degradation of intracellular retinoic acid-binding proteins (CRABP-I and CRABP-II) (Fig. 4a). Besides, bestatin-based PROTACs found efficiency towards other targets, such as AR, ${ }^{\mathbf{1 4}}$ $\mathrm{ER} \alpha,{ }^{15}$ TACC3 (ref. 16) and BCR-ABL. ${ }^{17}$ However, several limitations were observed, such as low potencies, off-target effects<smiles>COc1ccc(C2=NC(c3ccc(Cl)cc3)C(c3ccc(Cl)cc3)N2C(=O)N2CCN(CC(=O)NCCOCCOCCOCC(=O)Nc3ccc(OC[C@](C)(O)C(=O)Nc4ccc([N+](=O)[O-])c(C(F)(F)F)c4)cc3)CC2=O)c(OC(C)C)c1</smiles>

Fig. 3 An MDM2-based PROTAC (AR targeting). 
a.

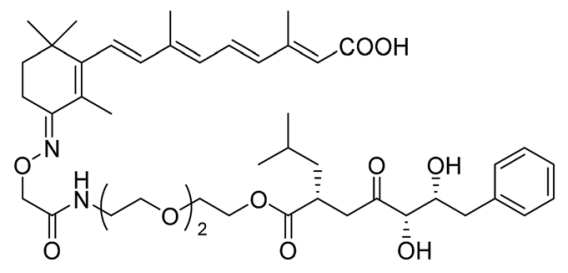

SNIPER-2 b.

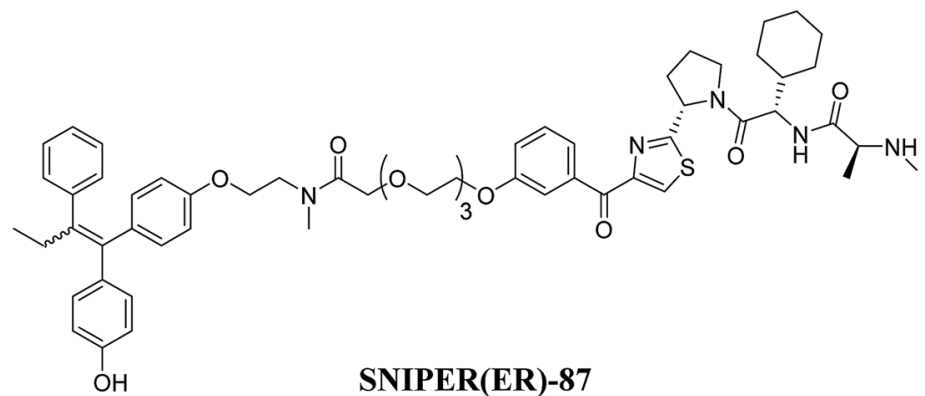

Fig. 4 (a) A clAP-based PROTAC (CRABPIl targeting), and (b) XIAP-based PROTAC (ER targeting).

and, especially, the self-degradation of cIAP1. To overcome these shortcomings, an IAP antagonist LCL161 was utilized to replace bestatin. ${ }^{\mathbf{1 8}}$ These new PROTACs, which recruit XIAP instead of cIAP1, successfully degrade targets at a nanomolar level (Fig. 4b).

2.2.3 CRBN-based PROTACs. The rapid development of PROTAC technology is attributed to the discovery of highaffinity small-molecule ligands in recent years, such as CRBN and VHL. In 2010, CRBN was identified as the primary target of thalidomide. Then, thalidomide and its derivatives were discovered to induce the degradation of IKZF1 and IKZF2 by binding to CRBN, thus resulting in the ubiquitination of targets. ${ }^{19,20}$ Given that thalidomide and its derivatives are capable of binding CRBN specifically, dBET1 was firstly synthesized to induce the degradation of BRD4, a bromodomain-containing protein. ${ }^{21}$ Over $85 \%$ of BRD4 was observed to degrade at a concentration of $100 \mathrm{nM}$ with dBET1 for $18 \mathrm{~h}$ in AML cells. The significant reduction of oncoproteins PIM1 and MYC was also noticed. Besides, dBET1 exhibited greater apoptosis induction than JQ1 both in AML cell-based assays and in a murine xenograft model of AML (Fig. 5a). Similar to dBET1, another PROTAC coupling OTX015 and pomalidomide, named ARV-825, was discovered to induce the almost-complete degradation of BRD4 at $10 \mathrm{nM}$ in $6 \mathrm{~h}$ (ref. 22) (Fig. 5b). Further studies indicated that ARV-825 is superior to the BRD4 inhibitors JQ1 and OTX015 for antiproliferation and apoptosis-induction. The discoveries of dBET1 and ARV-825 demonstrate that the proteolytic-induction of BRD4 by CRBN-based PROTACs is better than current small-molecule inhibitors. Moreover, Wang and his group attempted to optimize the PROTAC BET degrader: they used RX-37 (ref. 23) and QCA276 (ref. 24) as BRD4 ligands, successively. Surprisingly, the best compounds BETd260 and QCA570 were shown to effectively induce the 50\% degradation of $\mathrm{BRD} 4$ at a picomole level (the $\mathrm{DC}_{50}$ value). Ibrutinib is the first-in-class covalent BTK inhibitor for treating non-Hodgkin lymphoma, while it has also shown a variety of side effects due to inhibiting EGFR, ITK and TEC family kinases at the same time. The long-term administration of ibrutinib can cause C481S BTK mutation, which can thus lead to drug resistance. The PROTAC P13I, which adopts ibrutinib as a warhead and pomalidomide to recruit CRBN, was synthesized to target BTK degradation $^{25,26}$ (Fig. 5c). Surprisingly, P13I was observed to effectively degrade the C481S mutant BTK at $30 \mathrm{nM}$. Besides, P13I could significantly inhibit the proliferation of the $\mathrm{BTK}^{\mathrm{C} 481 \mathrm{~S}}$ DLBCL cell line. Moreover, several experiments testing the side effects were performed; P13I demonstrated no degradation and almost no inhibitory activity against EGFR, ITK or TEC, which emphasized that it is improbable that this BTK-targeting a.

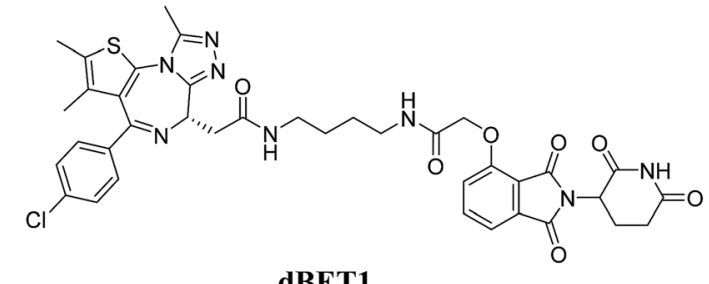

dBET1

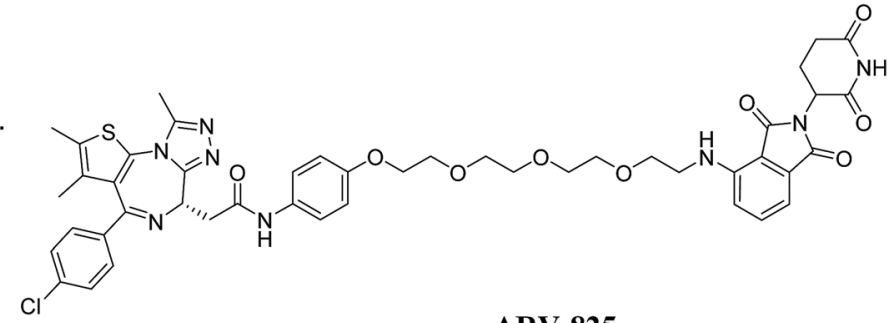

c.

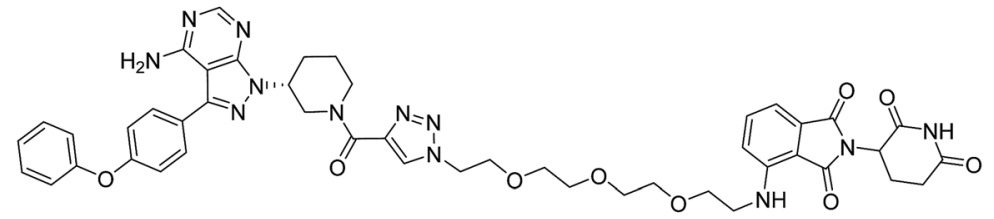

P13I

Fig. 5 CRBN-based PROTACs: (a) dBET1 (BRD4-targeting); (b) ARV-825 (BRD4-targeting); and (c) P13I (BTK-targeting). 
PROTAC would cause the aforementioned side effects of ibrutinib. This research indicates that PROTAC technology could be counted as a promising therapy for drug-resistant cancers. In addition to the above, CRBN-based PROTACs showed efficiencies against CDK9, ${ }^{27,28}$ BTK, ${ }^{29}$ HDAC6, ${ }^{30}$ ALK, ${ }^{31}$ BCR-ABL, ${ }^{32}$ Sirt2 (ref. 33) and $\mathrm{PI} 3 \mathrm{~K}^{34}$ that were better than the original inhibitors. In addition, these targets comprise nuclear receptors, transcriptional regulators and protein kinases, manifesting the universality of PROTACs as anticancer treatments. ${ }^{35}$

2.2.4 VHL-based PROTACs. Peptide PROTACs demonstrated the value of the VHL E3 ligase a long time ago. The development and success of VHL-based small-molecule PROTACs give credit to the discovery of potent inhibitors of VHL E3 ligase as a result of the efforts of C. M. Crews, A. Ciulli and their groups. ${ }^{36-39}$ With the ligand available, numerous PROTACs that recruit VHL have been reported. The $\mathrm{DC}_{50}$ value of the first VHLbased PROTAC targeted ERR $\alpha$ is about $100 \mathrm{nM}$. Significantly, this PROTAC that showed an efficacy in vivo that was about $40 \%$ of the ERR $\alpha$ level was degraded in murine heart, kidney and MDA-MB231 xenografted tumor models ${ }^{\mathbf{4 0 , 4 1}}$ (Fig. 6a). Based on this inspiring result, another PROTAC named PROTAC_RIPK2 effectively mediated the $95 \%$ knockdown of RIPK2 with maximal degradation abilities $\left(D_{\max }\right)$ at $10 \mathrm{nM}$ (ref. 40) (Fig. 6b). Moreover, an in vitro ubiquitination assay was established, which proved that one picomole of PROTAC_RIPK2 was shown to meditate 3.4 picomoles of RIPK2 ubiquitination. This result demonstrates why PROTACs can degrade the target protein with a $\mathrm{DC}_{50}$ value below the binding affinity of their target with the E3 ligase, and verifies the sub-stoichiometric catalytic nature of PROTACstheir key advantage over traditional small-molecule inhibitors ${ }^{\mathbf{4 0}}$.
Small-molecule VHL-based PROTACs have also been used to degrade BRD4. ARV-771, a picomolar VHL-based pan-BET degrader, which is under clinical study now, exerted efficiency against a CRPC mouse xenograft with activity much better than CRBN-based PROTAC dBET1 and ARV-825, revealing the capacity of PROTAC technology to combat solid tumors ${ }^{\mathbf{4 2}}$ (Fig. 6c). Another two BRD4-targeting PROTACs MZ1 and MZ2 both induce the degradation of BRD4 within $24 \mathrm{~h}$ (ref. 43) (Fig. 6d). Interestingly, these PROTACs induce the selective removal of BRD4 over BRD2 and BRD3, in contrast with dBET1 and ARV-825, indicating that the recruitment of different E3 ligases may lead to different selectivity profiles. Further studies utilizing the tyrosine kinase inhibitors bosutinib and dasatinib that target chimeric $\mathrm{BCR}-\mathrm{ABL}$ and $\mathrm{ABL}$ were carried out to compare VHL- and CRBN-based PROTAC in a head-to-head manner. ${ }^{32}$ Surprisingly, the selectivities of PROTACs depend on the identity of the inhibitor warhead as well as the recruited E3 ligase, despite target management. Moreover, the results indicated that the CRBN E3 ligase is more indiscriminate in its degradation profile against VHL, maybe owing to the flexible cullin 4A scaffold of the CRBN E3 ligase complex. ${ }^{44,45}$ In order to know more about the selective degradation of PROTACs, a promiscuous warhead Foretinib, which binds to 133 kinases, was chosen. ${ }^{46}$ Interestingly, the results indicated that both CRBN- and VHL-based PROTACs dramatically increase the degradation selectivities, in contrast with the parent warhead. Steric clashes that impact the stability of the ternary complex may influence the selectivity of the warhead. These studies inspire us as regards the potential of PROTAC to convert a nonselective or weak-affinity inhibitor into a selective a. $\mathrm{NC}$

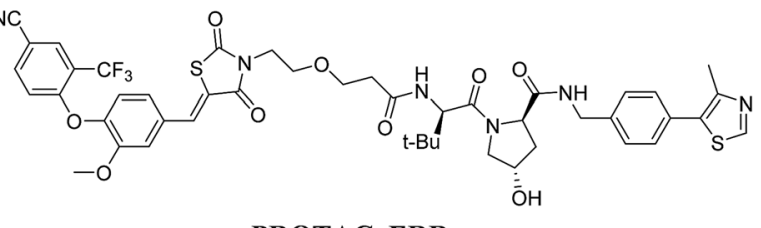

PROTAC_ERR

b.

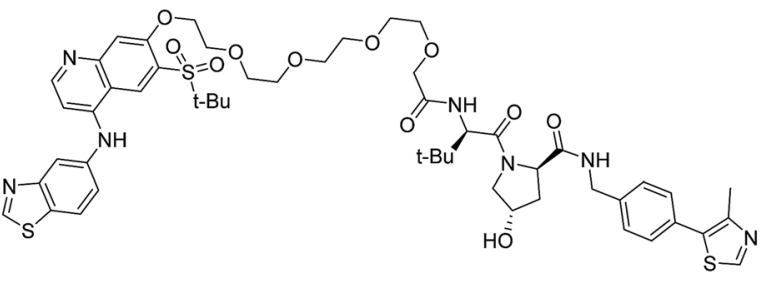

d.
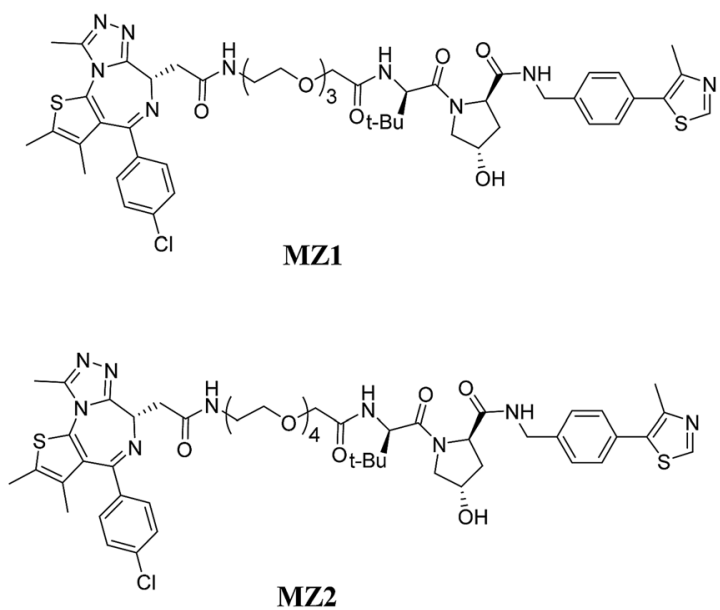

c.

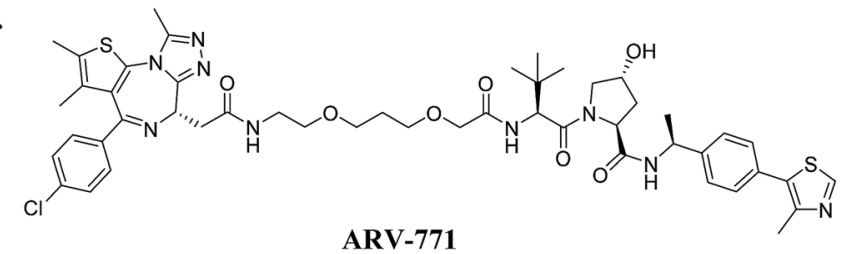

Fig. 6 VHL-based PROTACs: (a) PROTAC_ERR $\alpha$ (ERR $\alpha$-targeting); (b) PROTAC_RIPK2 (RIPK2-targeting); (c) ARV-771 (BRD4-targeting); and (d) MZ1/MZ2 (BRD4-targeting). 
degrader. To date, VHL-based PROTACs targeting DHODH ${ }^{47}$ AR, ${ }^{48,49}$ ER, ${ }^{50}$ TRIM24, ${ }^{51}$ Fak, ${ }^{52}$ FLT- $3,{ }^{53}$ ALK, ${ }^{54}$ p38 (ref. 55) and TANK $^{56}$ have also been reported.

\section{Challenges and opportunities}

\subsection{Expanding the library in use}

Though the prospect of PROTAC technology has attracted wide interest, some challenges still exist to be overcome. E3 ubiquitin ligases are a huge family with over 600 members, but only CRBN, VHL and IAP are presently widely used in PROTACs. A vast space remains to be excavated to allow more E3 ligases to be applied to PROTAC technology. Several obstacles lie in the way of developing new E3 ligases for PROTACs. Exploiting some small-molecule ligands with high affinities for one E3 ligase is paramount, since it is very difficult to develop inhibitors based on protein-protein interactions. Fortunately, over the past decades, some E3 ligase inhibitors have been discovered, which have the potential to be exploited as novel E3 ligase ligands for PROTACs.

The MDM2-based small-molecule PROTAC was discovered in 2008 , but it was not widely used because of its poor activity. ${ }^{12}$ This research lagged for 10 years until the well-reported study of MDM2 inhibitors. In 2018, C. M. Crews and his group reported A1874, a new MDM2-based PROTAC that comprises the BRD4 ligand JQ1 and an MDM2 antagonist idasanutlin with nanomolar potency. ${ }^{57}$ As MDM2-based PROTACs have the ability both to degrade target proteins and stabilize p53, A1874 was discovered to perform better in the anti-proliferation of many cancer cell lines with wide-type p53 than the corresponding VHL-based PROTAC. This research emphasizes the untapped potential of MDM2-based PROTACs and may expand the library of E3 ligases that can be used in PROTAC technology.

Keap1 is a member of the cullin3-based E3 ligase group, which directs its substrate Nrf2 towards degradation. Developing novel inhibitors that interrupt Keap1-Nrf2 PPI to enhance Nrf2 for the prevention of cancer is a feasible direction..$^{58-60} \mathrm{Z}$. Y. Jiang and his group developed a class of small-molecule inhibitors that target Keap1-Nrf2 PPI with nanomolar activities. $^{61-65}$ Besides, they also synthesized a Keap-dependent peptide PROTAC to knockdown Tau, ${ }^{66}$ indicating that a new class of small-molecule PROTACs based on the Keap1 E3 ligase can be expected and its development is only just a matter of time.

TRIM24 is a p53-induced E3-ubiquitin ligase that targets endogenous p53 for degradation. ${ }^{67,68}$ In 2015, J. Bennett and W. S. Palmer reported a series of TRIM24 inhibitors (e.g., IACS-7e and IACS-9571) with nanomolar potencies. ${ }^{69,70}$ A TRIM24 degrader was synthesized as a chemical probe for acute leukemia. ${ }^{51}$ Besides, IACS-9571 was reported in a patent to act as an E3 ligand to form a PROTAC for the degradation of EZH1/ EZH $2 .^{71}$

Interestingly, very similar to the discovery of the CRBN E3ubiquitin ligase as the primary target of thalidomide, ${ }^{19,20}$ anticancer sulfonamides (e.g., indisulam, E7820 and CQS) were reported to induce CAPER $\alpha$ (also known as RBM39) degradation by recruiting the DCAF15 E3 ligase. $^{72,73}$ Much evidence has proved that DCAF15 is the main target of anticancer sulfonamides. In addition, the E3 ligase DCAF15 belongs to the CUL4A/B family, just like $\mathrm{CRBN}^{74}$ and they show similar chemical modulation, which suggests that transforming sulfonamides into bifunctional PROTACs is a potential direction through recruiting the DCAF15 E3 ligase. ${ }^{75}$

\subsection{HOMO-PROTACs}

Except for recruiting E3 ligases to deplete target proteins, the over expression of several E3 ligases is often observed in various cancers; this is always correlated with chemo-resistance and poor prognoses. ${ }^{76}$ Inducing the degradation of the E3 ligase itself using PROTACs may be an effective strategy, which has thus produced the concept of homo-PROTACs. The first homoPROTAC, CM11, was constituted of two VHL inhibitors and one linker. ${ }^{77}$ This symmetric homodimerized compound induced the potent, rapid self-degradation of VHL in different cell lines. Like CM11, another CRBN-based homo-PROTAC was reported, which had the potent effect of antagonism with pomalidomide. $^{78}$

\subsection{Optimizing PROTAC technology}

In addition, various problems remain in existence before PROTAC technology can mature for clinical applications, such as off-target effects, in vivo metabolic stability, cellular permeability and large molecule weight. According to Lipinski's rule

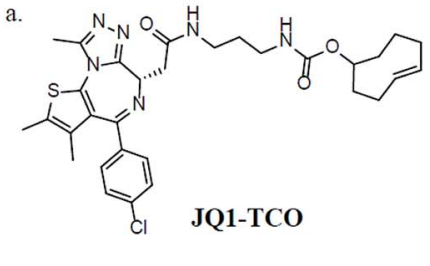

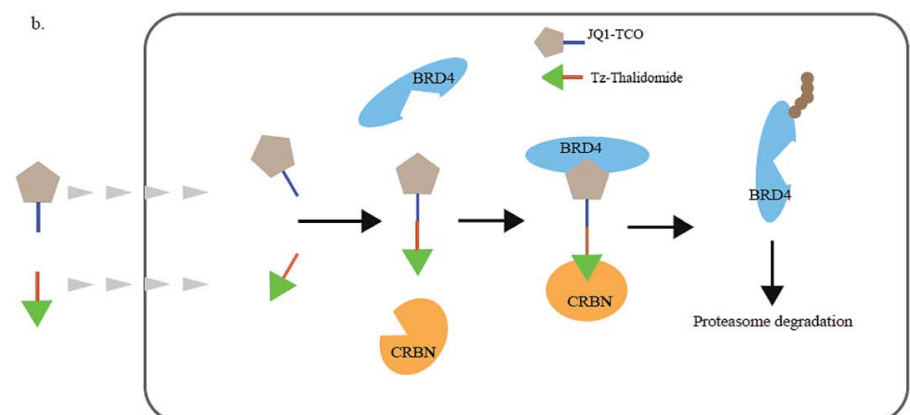

Fig. 7 (a) The structures of JQ1-TCO and Tz-thalidomide. (b) The mechanism of CLIPTACs. Two precursor molecules penetrate the cytomembrane in succession and form a CLIPTAC intracellularly, resulting in the proteasome degradation of BRD4. 


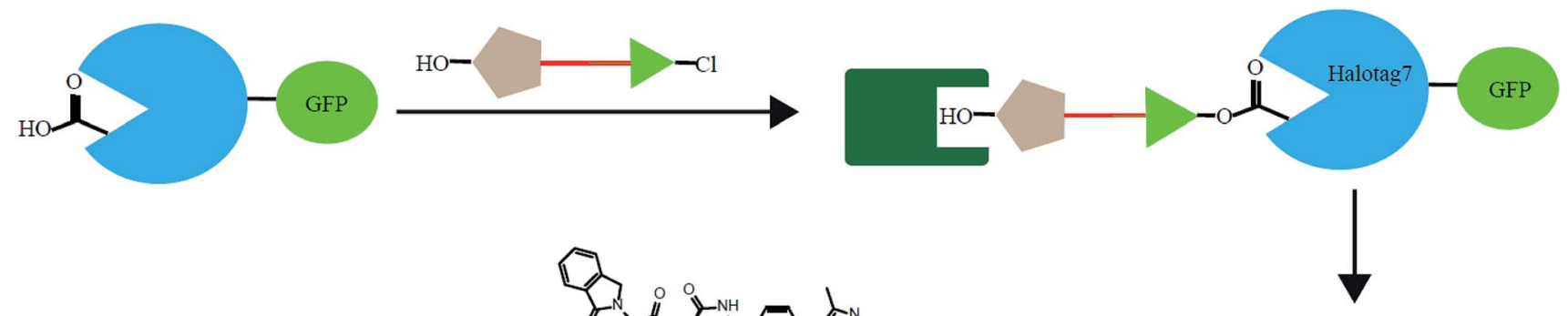

Proteasome degradation

HaloPROTAC-3

Fig. 8 A schematic diagram showing a HaloPROTAC. The HaloPTORAC utilizes the specific recognition by HaloTag7 of hexyl chloride tags and thus induces the degradation of HaloTag7-GFP fused proteins. This strategy simplifies the optimization of PROTACs.

of five, many efforts should be made to convert PROTAC molecules to be more "drug-like". Beyond that, it is hard to synthesize and optimize bifunctional molecules; these are also important obstacles in research and manufacture. On the bright side, many groups have tried to break through these barriers. An advanced technology called CLIPTAC (in-cell click-formed PROTAC) has been proposed, which separates the bifunctional molecule into two pairs, one pair with a tetrazine tagged thalidomide derivative and another pair with a trans-cyclooctene (TCO)-tagged ligand of the target protein in the cell ${ }^{79}$ (Fig. 7). In the concept of click chemistry, these two pairs are able to unite as one to form a PROTAC via a rapid reaction in a cell. Both of the pairs have lower molecular weights and better cell penetrability than the original PROTACs. This strategy can be expanded to induce the degradation of any target protein via an in-cell click-formed PROTAC. This click chemistry concept also provides a rapid synthesis method for PROTACs in vitro. ${ }^{80}$ Another technology named HaloPROTAC has been reported to target the degradation of intracellular GFP (green fluorescent protein)-HaloTag7 fused proteins, which greatly simplifies the optimization procedure of PROTACs in terms of finding appropriate linker lengths and linker attachment points ${ }^{81}$ (Fig. 8). Further, HaloTag7 was fused with different E3 ligases to degrade GFP, to evaluate the E3 ligase role in PROTAC technology. ${ }^{82}$

\section{Conclusions and future prospects}

In summary, PROTAC technology has shown significant improvements in recent decades, mainly from all-peptide to small-molecule compounds, and in terms of the cellular permeabilities, solubilities, stabilities and affinities of PROTACs. PROTAC technology shows many advantages over smallmolecule inhibitors, such as inducing the durative and fast depletion of target proteins and downstream signaling cascades, overcoming mutation-caused drug resistance, enhancing target selectivities and modifying potencies, and reducing dosage due to its sub-stoichiometric catalytic nature. Despite small molecules having achieved much success in being applied to classically druggable proteins in recent years, the most promising aspect of this technology is its potential to target "undruggable" proteins. Approximately $85 \%$ of the human proteome, such as transcription factors, scaffolding proteins, and non-enzyme proteins, has a lack of enzymatic activity or functional interactions. However, the event-driven nature of PROTACs permits their binding to any site of the target protein, instead of a stationary active site, thus driving these "undruggable" proteins to become pharmaceutically vulnerable.

Therefore, utilizing PROTACs to induce protein degradation has the potential of being a promising therapeutic strategy. To date, although most research into PROTACs has specially focused on applications to cancer therapy, the treatment of other diseases may benefit from this burgeoning technology. So far, only one PROTAC compound has been pushed to clinical trials recently. Therefore, the clinical outcome of PROTAC technology remains uncertain. Nevertheless, it is a long journey to advance PROTAC technology from the bench to the clinic. Overall, a large amount of effort is being made to develop PROTAC technology, and we are hoping for a bright future.

\section{Conflicts of interest}

The authors declare no conflicts of interest.

\section{Acknowledgements}

This work was supported by grants from the National Key R\&D Program of China (2018YFA0507001), the National Natural Science Foundation of China (81673304), and the Innovation program of the Shanghai Municipal Education Commission (2017-01-07-00-05-E00011).

\section{References}

1 S. Gu, D. Cui, X. Chen, X. Xiong and Y. Zhao, BioEssays, 2018, 40, 1700247.

2 M. Toure and C. M. Crews, Angew. Chem., Int. Ed. Engl., 2016, 55, 1966-1973. 
3 S. Dogan, R. Shen, D. C. Ang, M. L. Johnson, S. P. D'Angelo, P. K. Paik, E. B. Brzostowski, G. J. Riely, M. G. Kris, M. F. Zakowski and M. Ladanyi, Clin. Cancer Res., 2012, 18, 6169-6177.

4 E. Rozengurt, H. P. Soares and J. Sinnet-Smith, Mol. Cancer Ther., 2014, 13, 2477-2488.

5 E. Buck, A. Eyzaguirre, M. Rosenfeld-Franklin, S. Thomson, M. Mulvihill, S. Barr, E. Brown, M. O'Connor, Y. Yao, J. Pachter, M. Miglarese, D. Epstein, K. K. Iwata, J. D. Haley, N. W. Gibson and Q.-S. Ji, Cancer Res., 2008, 68, 8322-8332.

6 J. Spiegel, P. M. Cromm, G. Zimmermann, T. N. Grossmann and H. Waldmann, Nat. Chem. Biol., 2014, 10, 613-622.

7 A. C. Lai and C. M. Crews, Nat. Rev. Drug Discovery, 2017, 16, 101-114.

8 J. Salami and C. M. Crews, Science, 2017, 355, 1163-1167.

9 P. M. Cromm and C. M. Crews, Cell Chem. Biol., 2017, 24, 1181-1190.

10 K. M. Sakamoto, K. B. Kim, A. Kumagai, F. Mercurio, C. M. Crews and R. J. Deshaies, Proc. Natl. Acad. Sci. U. S. A., 2001, 98, 8554-8559.

11 J. S. Schneekloth, F. N. Fonseca, M. Koldobskiy, A. Mandal, R. Deshaies, K. Sakamoto and C. M. Crews, J. Am. Chem. Soc., 2004, 126, 3748-3754.

12 A. R. Schneekloth, M. Pucheault, H. S. Tae and C. M. Crews, Bioorg. Med. Chem. Lett., 2008, 18, 5904-5908.

13 Y. Itoh, M. Ishikawa, M. Naito and Y. Hashimoto, J. Am. Chem. Soc., 2010, 132, 5820-5826.

14 Y. Itoh, R. Kitaguchi, M. Ishikawa, M. Naito and Y. Hashimoto, Bioorg. Med. Chem., 2011, 19, 6768-6778.

15 K. Okuhira, Y. Demizu, T. Hattori, N. Ohoka, N. Shibata, T. Nishimaki-Mogami, H. Okuda, M. Kurihara and M. Naito, Cancer Sci., 2013, 104, 1492-1498.

16 N. Ohoka, K. Nagai, T. Hattori, K. Okuhira, N. Shibata, N. Cho and M. Naito, Cell Death Dis., 2014, 5, e1513.

17 Y. Demizu, N. Shibata, T. Hattori, N. Ohoka, H. Motoi, T. Misawa, T. Shoda, M. Naito and M. Kurihara, Bioorg. Med. Chem. Lett., 2016, 26, 4865-4869.

18 N. Ohoka, K. Okuhira, M. Ito, K. Nagai, N. Shibata, T. Hattori, O. Ujikawa, K. Shimokawa, O. Sano, R. Koyama, H. Fujita, M. Teratani, H. Matsumoto, Y. Imaeda, H. Nara, N. Cho and M. Naito, J. Biol. Chem., 2017, 292, 4556-4570.

19 J. Kronke, N. D. Udeshi, A. Narla, P. Grauman, S. N. Hurst, M. McConkey, T. Svinkina, D. Heckl, E. Comer, X. Y. Li, C. Ciarlo, E. Hartman, N. Munshi, M. Schenone, S. L. Schreiber, S. A. Carr and B. L. Ebert, Science, 2014, 343, 301-305.

20 T. Ito, H. Ando, T. Suzuki, T. Ogura, K. Hotta, Y. Imamura, Y. Yamaguchi and H. Handa, Science, 2010, 327, 1345-1350.

21 G. E. Winter, D. L. Buckley, J. Paulk, J. M. Roberts, A. Souza, S. Dhe-Paganon and J. E. Bradner, Science, 2015, 348, 13761381.

22 J. Lu, Y. Qian, M. Altieri, H. Dong, J. Wang, K. Raina, J. Hines, J. D. Winkler, A. P. Crew, K. Coleman and C. M. Crews, Chem. Biol., 2015, 22, 755-763.

23 B. Zhou, J. T. Hu, F. M. Xu, Z. Chen, L. C. Bai, E. FernandezSalas, M. Lin, L. Liu, C. Y. Yang, Y. J. Zhao, D. McEachern,
S. Przybranowski, B. Wen, D. X. Sun and S. M. Wang, J. Med. Chem., 2018, 61, 462-481.

24 C. Qin, Y. Hu, B. Zhou, E. Fernandez-Salas, C.-Y. Yang, L. Liu,

D. McEachern, S. Przybranowski, M. Wang, J. Stuckey,

J. Meagher, L. Bai, Z. Chen, M. Lin, J. Yang, D. N. Ziazadeh, F. Xu, J. Hu, W. Xiang, L. Huang, S. Li, B. Wen, D. Sun and S. Wang, J. Med. Chem., 2018, 61, 6685-6704.

25 Y. Sun, X. Zhao, N. Ding, H. Gao, Y. Wu, Y. Yang, M. Zhao, J. Hwang, Y. Song, W. Liu and Y. Rao, Cell Res., 2018, 28, 779-781.

26 A. D. Buhimschi, H. A. Armstrong, M. Toure, S. JaimeFigueroa, T. L. Chen, A. M. Lehman, J. A. Woyach, A. J. Johnson, J. C. Byrd and C. M. Crews, Biochemistry, 2018, 57, 3564-3575.

27 C. M. Robb, J. I. Contreras, S. Kour, M. A. Taylor, M. Abid, Y. A. Sonawane, M. Zahid, D. J. Murry, A. Natarajan and S. Rana, Chem. Commun., 2017, 53, 7577-7580.

28 C. M. Olson, B. Jiang, M. A. Erb, Y. Liang, Z. M. Doctor, Z. Zhang, T. Zhang, N. Kwiatkowski, M. Boukhali, J. L. Green, W. Haas, T. Nomanbhoy, E. S. Fischer, R. A. Young, J. E. Bradner, G. E. Winter and N. S. Gray, Nat. Chem. Biol., 2018, 14, 163-170.

29 A. Zorba, C. Nguyen, Y. R. Xu, J. Starr, K. Borzilleri, J. Smith, H. Y. Zhu, K. A. Farley, W. D. Ding, J. Schiemer, X. D. Feng, J. S. Chang, D. P. Uccello, J. A. Young, C. N. Garcia-Irrizary, L. Czabaniuk, B. Schuff, R. Oliver, J. Montgomery, M. M. Hayward, J. Coe, J. S. Chen, M. Niosi, S. Luthra, J. C. Shah, A. El-Kattan, X. Y. Qiu, G. M. West, M. C. Noe, V. Shanmugasundaram, A. M. Gilbert, M. F. Brown and M. F. Calabrese, Proc. Natl. Acad. Sci. U. S. A., 2018, 115, E7285-E7292.

30 K. Yang, Y. Song, H. Xie, H. Wu, Y.-T. Wu, E. D. Leisten and W. Tang, Bioorg. Med. Chem. Lett., 2018, 28, 2493-2497.

31 C. Zhang, X.-R. Han, X. Yang, B. Jiang, J. Liu, Y. Xiong and J. Jin, Eur. J. Med. Chem., 2018, 151, 304-314.

32 A. C. Lai, M. Toure, D. Hellerschmied, J. Salami, S. JaimeFigueroa, E. Ko, J. Hines and C. M. Crews, Angew. Chem., Int. Ed., 2016, 55, 807-810.

33 M. Schiedel, D. Herp, S. Hammelmann, S. Swyter, A. Lehotzky, D. Robaa, J. Olah, J. Ovadi, W. Sippl and M. Jung, J. Med. Chem., 2018, 61, 482-491.

34 W. Li, C. Gao, L. Zhao, Z. Yuan, Y. Chen and Y. Jiang, Eur. J. Med. Chem., 2018, 151, 237-247.

35 Y. Zou, D. Ma and Y. Wang, Cell Biochem. Funct., 2019, 37, 21-30.

36 D. L. Buckley, I. Van Molle, P. C. Gareiss, H. S. Tae, J. Michel, D. J. Noblin, W. L. Jorgensen, A. Ciulli and C. M. Crews, J. Am. Chem. Soc., 2012, 134, 4465-4468.

37 C. Galdeano, M. S. Gadd, P. Soares, S. Scaffidi, I. Van Molle, I. Birced, S. Hewitt, D. M. Dias and A. Ciulli, J. Med. Chem., 2014, 57, 8657-8663.

38 P. Soares, M. S. Gadd, J. Frost, C. Galdeano, L. Ellis, O. Epemolu, S. Rocha, K. D. Read and A. Ciulli, J. Med. Chem., 2018, 61, 599-618.

39 X. Lucas, I. Van Molle and A. Ciulli, J. Med. Chem., 2018, 61, 7387-7393. 
40 D. P. Bondeson, A. Mares, I. E. Smith, E. Ko, S. Campos, A. H. Miah, K. E. Mulholland, N. Routly, D. L. Buckley, J. L. Gustafson, N. Zinn, P. Grandi, S. Shimamura, G. Bergamini, M. Faelth-Savitski, M. Bantscheff, C. Cox, D. A. Gordon, R. R. Willard, J. J. Flanagan, L. N. Casillas, B. J. Votta, W. den Besten, K. Famm, L. Kruidenier, P. S. Carter, J. D. Harling, I. Churcher and C. M. Crews, Nat. Chem. Biol., 2015, 11, 611-617.

41 R. J. Deshaies, Nat. Chem. Biol., 2015, 11, 634-635.

42 K. Raina, J. Lu, Y. Qian, M. Altieri, D. Gordon, A. M. Rossi, J. Wang, X. Chen, H. Dong, K. Siu, J. D. Winkler, A. P. Crew, C. M. Crews and K. G. Coleman, Proc. Natl. Acad. Sci. U. S. A., 2016, 113, 7124-7129.

43 M. Zengerle, K. H. Chan and A. Ciulli, ACS Chem. Biol., 2015, 10, 1770-1777.

44 E. S. Fischer, A. Scrima, K. Boehm, S. Matsumoto, G. M. Lingaraju, M. Faty, T. Yasuda, S. Cavadini, M. Wakasugi, F. Hanaoka, S. Iwai, H. Gut, K. Sugasawa and N. H. Thomae, Cell, 2011, 147, 1024-1039.

45 J. Liu and R. Nussinov, J. Biol. Chem., 2011, 286, 4093440942.

46 D. P. Bondeson, B. E. Smith, G. M. Burslem, A. D. Buhimschi, J. Hines, S. Jaime-Figueroa, J. Wang, B. D. Hamman, A. Ishchenko and C. M. Crews, Cell Chem. Biol., 2018, 25, 78-87.e5.

47 J. T. Madak, C. R. Cuthbertson, W. Chen, H. D. Showalter and N. Neamati, Chem. - Eur. J., 2017, 23, 13875-13878.

48 T. K. Neklesa, M. Jin, A. P. Crew, A. K. Rossi, R. R. Willard, H. Dong, K. Siu, J. Wang, D. A. Gordon, X. Chen, C. Ferraro, C. M. Crews, K. Coleman and J. D. Winkler, J. Clin. Oncol., 2016, 34(suppl. 2), 267.

49 X. Han, C. Wang, C. Qin, W. Xiang, E. Fernandez-Salas, C.-Y. Yang, M. Wang, L. Zhao, T. Xu, K. Chinnaswamy, J. Delproposto, J. Stuckey and S. Wang, J. Med. Chem., 2019, 62, 941-964.

50 J. Hu, B. Hu, M. Wang, F. Xu, B. Miao, C.-Y. Yang, M. Wang, Z. Liu, D. F. Hayes, K. Chinnaswamy, J. Delproposto, J. Stuckey and S. Wang, J. Med. Chem., 2019, 1420-1442.

51 L. N. Gechijian, D. L. Buckley, M. A. Lawlor, J. M. Reyes, J. Paulk, C. J. Ott, G. E. Winter, M. A. Erb, T. G. Scott, M. Xu, H. S. Seo, S. Dhe-Paganon, N. P. Kwiatkowski, J. A. Perry, J. Qi, N. S. Gray and J. E. Bradner, Nat. Chem. Biol., 2018, 14, 405-412.

52 P. M. Cromm, K. T. G. Samarasinghe, J. Hines and C. M. Crews, J. Am. Chem. Soc., 2018, 140, 17019-17026.

53 G. M. Burslem, J. Song, X. Chen, J. Hines and C. M. Crews, J. Am. Chem. Soc., 2018, 140, 16428-16432.

54 C. H. Kang, D. H. Lee, C. O. Lee, J. D. Ha, C. H. Park and J. Y. Hwang, Biochem. Biophys. Res. Commun., 2018, 505, 542-547.

55 B. E. Smith, S. L. Wang, S. Jaime-Figueroa, A. Harbin, J. Wang, B. D. Hamman and C. M. Crews, Nat. Commun., 2019, 10, 131.

56 A. P. Crew, K. Raina, H. Q. Dong, Y. M. Qian, J. Wang, D. Vigil, Y. V. Serebrenik, B. D. Hamman, A. Morgan, C. Ferraro, K. Siu, T. K. Neklesa, J. D. Winkler,
K. G. Coleman and C. M. Crews, J. Med. Chem., 2018, 61, 583-598.

57 J. Hines, S. Lartigue, H. Dong, Y. Qian and C. M. Crews, Cancer Res., 2019, 79, 251-262.

58 T. Suzuki, H. Motohashi and M. Yamamoto, Trends Pharmacol. Sci., 2013, 34, 340-346.

59 M. C. Jaramillo and D. D. Zhang, Genes Dev., 2013, 27, 21792191.

60 M. C. Lu, J. A. Ji, Z. Y. Jiang and Q. D. You, Med. Res. Rev., 2016, 36, 924-963.

61 Z. Y. Jiang, M. C. Lu, L. L. Xu, T. T. Yang, M. Y. Xi, X. L. Xu, X. K. Guo, X. J. Zhang, Q. D. You and H. P. Sun, J. Med. Chem., 2014, 57, 2736-2745.

62 Z. Y. Jiang, M. C. Lu and Q. D. You, J. Med. Chem., 2016, 59, 10837-10858.

63 M. Lu, H. S. Zhou, Q. D. You and Z. Jiang, J. Med. Chem., 2016, 59, 7305-7310.

64 M. C. Lu, S. J. Tan, J. A. Ji, Z. Y. Chen, Z. W. Yuan, Q. D. You and Z. Y. Jiang, ACS Med. Chem. Lett., 2016, 7, 835-840.

65 H.-P. Sun, Z.-Y. Jiang, M.-Y. Zhang, M.-C. Lu, T.-T. Yang, Y. Pan, H.-Z. Huang, X.-J. Zhang and Q.-d. You, Med. Chem. Commun., 2014, 5, 93-98.

66 M. Lu, T. Liu, Q. Jiao, J. Ji, M. Tao, Y. Liu, Q. You and Z. Jiang, Eur. J. Med. Chem., 2018, 146, 251-259.

67 K. Allton, A. K. Jain, H. M. Herz, W. W. Tsai, S. Y. Jung, J. Qin, A. Bergmann, R. L. Johnson and M. C. Barton, Proc. Natl. Acad. Sci. U. S. A., 2009, 106, 11612-11616.

68 A. K. Jain, K. Allton, A. D. Duncan and M. C. Barton, Mol. Cell. Biol., 2014, 34, 2695-2709.

69 J. Bennett, O. Fedorov, C. Tallant, O. Monteiro, J. Meier, V. Gamble, P. Savitsky, G. A. Nunez-Alonso, B. Haendler, C. Rogers, P. E. Brennan, S. Muller and S. Knapp, J. Med. Chem., 2016, 59, 1642-1647.

70 W. S. Palmer, G. Poncet-Montange, G. Liu, A. Petrocchi, N. Reyna, G. Subramanian, J. Theroff, A. Yau, M. KostAlimova, J. P. Bardenhagen, E. Leo, H. E. Shepard, T. N. Tieu, X. Shi, Y. Zhan, S. Zhao, M. C. Barton, G. Draetta, C. Toniatti, P. Jones, M. Geck Do and J. N. Andersen, J. Med. Chem., 2016, 59, 1440-1454.

71 J. Jin, WO2018081530A1, 2018.

72 T. Han, M. Goralski, N. Gaskill, E. Capota, J. Kim, T. C. Ting, Y. Xie, N. S. Williams and D. Nijhawan, Science, 2017, 356, eaal3755.

73 T. Uehara, Y. Minoshima, K. Sagane, N. H. Sugi, K. O. Mitsuhashi, N. Yamamoto, H. Kamiyama, K. Takahashi, Y. Kotake, M. Uesugi, A. Yokoi, A. Inoue, T. Yoshida, M. Mabuchi, A. Tanaka and T. Owa, Nat. Chem. Biol., 2017, 13, 675-680.

74 J. Cheng, J. Guo, B. J. North, K. Tao, P. Zhou and W. Wei, Biochim. Biophys. Acta, Rev. Cancer, 2018, 1871, 138-159.

75 G. E. Winter, Nat. Chem. Biol., 2017, 13, 575-576.

76 Y. Sun, Neoplasia, 2006, 8, 645-654.

77 C. Maniaci, S. J. Hughes, A. Testa, W. Chen, D. J. Lamont, S. Rocha, D. R. Alessi, R. Romeo and A. Ciulli, Nat. Commun., 2017, 8, 830. 
78 C. Steinebach, S. Lindner, N. D. Udeshi, D. C. Mani, H. Kehm, S. Kopff, S. A. Carr, M. Gutschow and J. Kronke, ACS Chem. Biol., 2018, 13, 2771-2782.

79 H. Lebraud, D. J. Wright, C. N. Johnson and T. D. Heightman, ACS Cent. Sci., 2016, 2, 927-934.

80 R. P. Wurz, K. Dellamaggiore, H. Dou, N. Javier, M. C. Lo, J. D. McCarter, D. Mohl, C. Sastri, J. R. Lipford and V. J. Cee, J. Med. Chem., 2018, 61, 453-461.
81 D. L. Buckley, K. Raina, N. Darricarrere, J. Hines, J. L. Gustafson, I. E. Smith, A. H. Miah, J. D. Harling and C. M. Crews, ACS Chem. Biol., 2015, 10, 1831-1837.

82 P. Ottis, M. Toure, P. M. Cromm, E. Ko, J. L. Gustafson and C. M. Crews, ACS Chem. Biol., 2017, 12, 2570-2578. 